CLINICAL ETHICS

\title{
Just allocation and team loyalty: a new virtue ethic for emergency medicine
}

J Girod, A W Beckman

See end of article for

authors' affiliations

J Med Ethics 2005;31:567-570. doi: 10.1136/jme.2004.009332

\section{Correspondence to:} Jennifer Girod, $618 \mathrm{E}$ Third Street, Bloomington IN 47405, USA; igirod@indiana.edu

Received 10 May 2004 In revised form 14 September 2004 Accepted for publication 17 December 2004

\begin{abstract}
When traditional virtue ethics is applied to clinical medicine, it often claims as its goal the good of the individual patient, and focuses on the dyadic relationship between one physician and one patient. An alternative model of virtue ethics, more appropriate to the practice of emergency medicine, will be outlined by this paper. This alternative model is based on the assumption that the appropriate goal of the practice of emergency medicine is a team approach to the medical wellbeing of individual patients, constrained by the wellbeing of the patient population served by a particular emergency department. By defining boundaries and using the key virtues of justice and team loyalty, this model fits emergency practice well and gives care givers the conceptual clarity to apply this model to various conflicts both within the department and with those outside the department.
\end{abstract}

$\mathrm{T}$ he idea of teaching physicians to be virtuous has regained a sense of importance and urgency in the current health care environment. ${ }^{1-4}$ Rather than delineating specific rules and policies to safeguard patients, virtue theorists focus on the development of habits and dispositions of physicians, in essence arguing that those who are trained well will naturally make virtuous decisions in the constantly changing clinical environment of modern medicine. Discussions of virtue are found in much of the medical professionalism literature across medical disciplines, including the specialty of emergency medicine (EM)..$^{5-9}$

Virtue theory can indeed be useful in many clinical practice settings, including EM. The theory must, however, be expanded in a way that coheres with the current health care delivery system in the USA. Physician workloads are greater than ever before, care is reviewed and managed by bureaucrats within and outside the hospital, and patient care is shared by a growing team of variously trained health care professionals. The practice of EM serves as the most obvious example of a medical specialty whose practitioners must find a way to manage and prioritise personal, patient, family, community, and staff needs in a virtuous manner.

This paper will outline an alternative model of virtue ethics more appropriate to the practice of EM. This model is based on the assumption that the appropriate goal of the practice of emergency medicine is the medical wellbeing of individual patients constrained by the good of the patient population served by a particular emergency department (ED). This model also depends on loyal relationships among members of the health care team as both instrumental to the delivery of quality care and as a good in itself. By defining boundaries and using the key virtues of justice and team loyalty, this model fits EM practice well and gives care givers the conceptual clarity to apply this model to various conflicts both within the department and with those outside the department.

A brief summary of the traditional conception of physician virtue in medical professionalism literature in general and EM professionalism literature in particular is necessary before describing in more detail an alternative model of virtue ethics. Renowned philosopher and physician Edmund Pellegrino is perhaps the most notable proponent of virtue theory in medicine. Following Aristotle's teleological model, he contends that the virtue of the physician is determined by the end or telos of medicine, which can be defined as the "patient's good". ${ }^{10}$

The good the patient seeks is to be healed-to be restored to his prior, or to a better, state of function, to be made "whole" again. The immediate end of medicine is not simply a technically proficient performance but the use of that performance to attain a good end-the good of the patient-his medical or biomedical good to the extent possible but also his good as he the patient perceives it; his good as a human person who can make his own life plan, and his good as a person with a spiritual destiny if this is his belief. It is the sensitive balancing of these senses of the patient's good which the virtuous physician pursues to perfection.

This conception is notably dyadic; that is, it focuses on the relationship between two individuals, the physician and a single patient. This restriction of the professional's ethical focus to his or her own character and the good of a single patient is an attempt to draw professional boundaries in a way that excludes the illegitimate interests of third parties. What is purposefully excluded in this account of virtue is the broader context of care givers and patients. Pellegrino would not wish any physician to be concerned with financial matters, other patients who may be waiting to be seen, or duties to other providers.

Professionalism literature in EM has largely followed the dyadic virtue account advocated by Pellegrino. As Michelle A Finkel and James G Adams assert: "The patient/physician relationship is grounded in the mutual belief that the actions of the physician are directed at the wellbeing of the individual patient". ' They argue therefore that physicians must suspend their own self interest, remain honest, and adhere to professional standards, thereby practising medicine in a way that preserves patient trust and serves the patient's best medical interest. ${ }^{9}$ This is good advice in the context of dilemmas affecting financial issues. In other matters that affect the daily clinical practice of EM, reference to the patient/physician dyad is insufficient. How, for example,

Abbreviations: ED, emergency department; $E M$, emergency medicine; $E P$, emergency physician 
should a patient's non-medical needs be handled? What should happen when a physician and nurse disagree about what medical treatment is best for a patient? What if the physician and nurse disagree about matters of moral judgment? What if there is a medical disagreement between the emergency physician (EP) and a surgical consultant?

The dyadic conception of virtue is overly narrow in the practice of EM. Emergency physicians often take care of several patients simultaneously, and busy EDs may see over 100000 patients per year. To care for anyone effectively in this environment requires wise decisions by care givers about the allocation of resources. Those resources are not primarily financial, but more often involve limited time, what may be an insufficient number of good patient rooms, and care giver energy. These are issues of justice. In addition, it is inappropriate to focus on the physician as an isolated moral actor in this context. Every patient encounter includes other health care providers: making decisions or policies without taking other providers into account is disrespectful and destructive of working relationships, and will ultimately impact negatively on the patient population the ED is designed to serve.

\section{DISCUSSION}

There are two crucial questions to answer when proposing an alternative ethic for emergency medicine: what constitutes the appropriate telos for an emergency department and what kind of virtue ethic is appropriate?

\section{Medical wellbeing as telos}

First, in theory and in practice the most ethically justifiable goal of an ED is to serve the medical needs of each individual patient as long as that care does not jeopardise the wellbeing of the ED's patient population. This patient population is a nebulous entity of present and future patients. This designation is broader than that of Pellegrino, Finkel, Adams, and others who stipulate the medical and psychical good of the individual patient as the goal of medicine, without reference to their place in the larger patient population. In reality, the goal of the ED system at any one time is not to serve the interest of one patient in isolation from concerns of other patients. Both the physical layout of the ED and its staffing patterns are designed to serve a group of patients simultaneously. This means there are competing obligations that physicians and others must consider when treating the individual patient, and limits must be set on what each individual patient may expect if EM is to be practised competently and justly.

How does changing the telos of EM from the best interests of the individual to the medical needs of the patient population change the treatment that individuals will receive? What practical differences will it make, and why are they justified? Competent, efficient care will remain paramount for each patient, but differences will arise when duties to provide "holistic" or humanistic care, or to respond to non-medical needs of patients, are considered.

The EP practises virtue when he or she provides clinically competent care for every individual who presents to the emergency department. Efficiency is the basis for all good care in the ED. Without it, people may leave without treatment and never receive care. If they stay, but wait a long period of time, they may receive delayed diagnosis and treatment for life threatening medical problems. Once patients are seen by their care givers, they should receive thorough medical evaluation by trained professionals who will determine whether they have a medical condition. If they do have a medical condition, they deserve state of the art care to correct the problem in the ED or to stabilise the problem until a specialist can be seen. Because quality care often mandates follow up, the EP or other providers should make sure that the patient understands where to receive follow up care. The three skills mentioned here: efficiency, diagnostic skills, and clinical management skills are three crucial components of offering clinically effective and competent care.

When do duties to the population as a whole supersede individual needs? The first and most obvious thing to state is that those individuals who are the sickest MUST receive care first in the ED. Good clinical care depends on physicians and nurses making educated and appropriate decisions about this issue. Of course, individual patients may suffer because of this basic principle. They may not be seen promptly, and once they have been seen they may have to wait a long time for the physicians and nurses to address their complaints. This is not optimal for any one patient, but it is necessary to make sure that individual members of the patient population receive life saving medical treatment when necessary.

One feature of care that receives diminished attention in the patient population model is the focus on the interpersonal relationship between physician and patient. Professionalism literature based on the traditional dyadic vision of the virtuous physician calls for kindness, concern, patience, and human respect for all patients, regardless of the patient's behaviour. By way of contrast, this model contends that the appropriate affective response will vary from one provider to the next, and can only be determined by how it impacts on clinical efficiency. A male EP with misogynist sentiments who gives clinically inferior care to a woman lacks virtue because he fails to provide competent care to that individual patient. The EP who sits at the bedside of a patient for two hours compassionately listening to his or her concerns also lacks virtue because the EP's obligations to other patients are thereby neglected. Kind and considerate treatment in this alternative virtue model is not valued because it is associated with high moral character, but because it will often positively impact patient understanding and compliance. The most virtuous physician in the patient population virtue model is one who, when busy, modulates his or her behaviour toward each patient in a way that will quickly and effectively advance the patient's medical best interest.

A second reason for focusing on the medical needs of the patient population as the goal of emergency medicine is that individuals who come to the ED seeking non-medical care are not considered the professional responsibility of the care givers who work there. (For the purposes of this essay, perceived medical needs are understood to be physical or psychiatric complaints for which an individual seeks a physician's clinical assessment, diagnosis, and appropriate treatment. It is possible that the physician will conclude that a medical problem does not exist, but that does not mean the patient does not require assessment. Non-medical needs are those needs perceived by an individual for which a physician's expertise is not needed and for which there is no medically therapeutic response.) Examples of individuals with non-medical needs include those seeking shelter, food, human contact and attention, and addictive substances for consumption or sale. To be clear, individuals with drug addictions who are seeking medical care and referrals for treatment for addiction are properly considered a part of the ED patient population; only those individuals seeking nonmedical care are excluded. This is a small but intensely time consuming portion of an ED's clinical burden. As stated previously, all individuals who come to the ED deserve thorough evaluation, but if, after that evaluation, the physician determines that there is no medical need to be met, those individuals fall outside the patient population properly served by professionals in the ED.

Satisfying non-medical needs in the ED hinders the goal of providing appropriate medical care to the ED patient population. Giving individuals food, shelter, or addictive 
substances reinforces habits that encourage individuals to use the ED for non-medical purposes, and that habit leads to a decreased level of care for others by draining a variety of the shared resources in the ED, including patient rooms, time, and care giver energy. It is to be hoped that over time the presence of these individuals will decrease if care givers consistently refuse to satisfy their non-medical needs.

Although individuals with non-medical needs should not be allocated patient examination rooms, this is not to say that all of these people must necessarily be evicted from the waiting room. Each ED should assess its own space constraints and available security personnel to handle, for instance, homeless people in harsh weather conditions. If an ED can help individuals avoid medical problems without hindering its own operation, then it would be just, compassionate, and financially worthwhile to do so.

The claim that EDs should serve only individuals who have medical needs is highly controverted within the EM community. Many argue that the ED serves not only as a medical safety net, but also as a social safety net, providing help or referrals for help for individuals who need food, housing, clothing, education, or work. (An anonymous reviewer also makes this point in relation to our paper. $)^{11}$ While these are all worthy social goals, there is no compelling reason to address them in the ED. Society should work to solve social problems caused by poverty and inadequate education, both of which may affect individuals who present to an ED. There is, however, no reason to think that EPs, either in the hospital or outside of it, are particularly well equipped to deal with these complex problems.

\section{TEAM INTEGRITY}

The misconceived telos of EM as the good of the individual patient without constraints constitutes one deficiency of the traditional dyadic professionalism model. The second reason this model is deficient in EM is because the physician always works with others. Part of serving the patient population effectively and virtuously depends upon virtuous and loyal relationships between the staff members who share in its care. We must move away from the dyadic theory common in professionalism literature and toward a team oriented virtue ethic. Effective advocacy for this patient population depends, however, on an accurate description of who is and who is not part of the health care team.

The appropriate designation of the morally relevant health care team is much broader than an individual physician, and includes the physicians, medical students (and residents in some settings, ) nurses, allied health professionals, administrative staff, and security staff employed by a particular ED. Effective advocacy for the patient population makes it necessary to designate insurance companies and health maintenance organisations (HMOs), physicians, and other health care providers in other areas of the hospital, and health care providers at other hospitals outside the health care team, because they do not share the same primary mission of serving the medical needs of that particular ED population.

What practical ramifications does drawing the boundaries of the professional team in this way have on patient care and interpersonal relationships among health care providers?

Team integrity is critical to achieve two discrete goods in the ED. First, the team must function well to provide efficient and competent care for the patient population. All of those working in a particular ED ostensibly share the goal of providing effective care in a particular kind of environment. Maintaining good working relationships is therefore instrumental to a goal that all members of the team share. Second, good working relationships are valued for their own sakes. Unlike the patient/physician relationship, provider relationships exist over time and have the potential to be a source of human trust and mutual support.

\section{CASES}

Conflicts within the team occur most frequently between a physician and a nurse in the ED. Two examples follow, one related to a conflict over a factual matter, and one to a matter more clearly concerning moral judgment or policy.

Consider this case of a conflict over a medical matter. A patient in the ED has pain due to a sickle cell crisis. The physician writes an order for $10 \mathrm{mg}$ of morphine to be given intravenously to this patient. The nurse approaches the physician and reports that he gave $4 \mathrm{mg}$ to the patient. When the physician asks why, he reports that he does not feel comfortable giving $10 \mathrm{mg}$ intravenously. The physician explains that the therapeutic dosage (the dosage expected to relieve pain) is $.1 \mathrm{mg} / \mathrm{kg}$, and that a $6-10 \mathrm{mg}$ dose is appropriate for an average size adult.

Three responses are common, but morally deficient, in this kind of scenario: 1) the physician does not argue with the nurse, but suggests a second dose of $4 \mathrm{mg}$ in thirty minutes; 2) the physician finds another nurse to give the dose, or 3) the physician administers the medication personally. Why are these responses inadequate? In all three cases the goals of physician and nurse cohere: both wish to provide effective and safe care for the patient.

The first response of allowing the nurse to divide and therefore decrease the dosage maintains peace with the nurse, but at the expense of their shared goal of helping the patient. It therefore does not accomplish the goal of either provider. In the short run it saves the physician time, although the patient population (and the individual patient) is better served both medically and in terms of length of visit if these issues are faced squarely and settled. The interaction therefore fails in virtue because it is not conducive to the telos of the practice of EM. In addition, all care givers taking care of this patient are legally responsible for treating his pain. If the physician and nurse share in moral deficiency, however well intentioned, they may also share legal liability.

In the second two scenarios the patient receives the therapeutic dose of pain medicine, but at the expense of the physician/nurse relationship. The nurse perceives that he took a conscientious stand in a moral disagreement and that the physician acted wrongly and in a disloyal manner by overriding the nurse's objections. The physician feels that the nurse has a knowledge deficit and is stubborn. The result of that kind of interaction between physician and nurse is a breakdown of relationship, respect, and loyalty.

Whenever possible, this kind of disagreement, which is factual and not moral, should be addressed proactively through interdisciplinary education. This could consist of several short conferences or by finding the appropriate medical literature and distributing it with an explanation and a person to contact with questions. When it cannot be addressed proactively, therapeutic doses of pain medicine should always be given, but the situation should be handled with delicacy and respect for the ongoing relationships between physicians and nurses. Short conversations between the physician and the nurse after such an occurrence can give both sides an opportunity to vent their feelings and gain an understanding of the other person's position.

Conflicts between physicians and nurses also occur over matters of moral judgment. Consider a case of a patient who comes to the ED complaining of chest pain, but the nurse reports to the physician that she believes he is only looking for food. She exhorts the physician not to feed the patient. After a thorough medical evaluation, the physician concludes that the patient does not have a medical problem. It is possible the physician will agree with the nurse and refuse to feed the patient. It is also possible that the physician will feed the patient over the nurse's objection due to time constraints or because he or she finds the nurse's suggestion unkind. In 
this case there is no agreed upon medical goal for the patient, and justice in the ED precludes the meeting of non-medical needs. Although time may be spared in the short term, reinforcing these habits would encourage visits in the future.

How should the physician respond to the nurse in this situation? Whether the nurse seems unkind or bitter in general should not affect decision making in individual instances. In this case, the physician who unilaterally disregards the advice of the nurse and feeds patients over nursing staff objections is overbearing, and any intention of inspiring virtue by doing this is bound to back fire. The long term physician/nurse relationship will suffer, and care for the patient population will suffer as well. Just as one provider cannot provide the medical care necessary for patients without the help of others, so that provider can't "exhibit virtue" over the protestations of other members of the team without demeaning other members and weakening the bonds that make unified action possible.

There are good reasons based on justice to oppose feeding individuals without medical complaints. Physicians should respect the moral intuitions of nurses who argue that doing so is a poor use of ED resources. All education, decisions, and policies about this issue and similar ones should include both physicians and nurses.

Doctors from other services within the hospital, and physicians and insurance managers from outside the hospital must be designated as outside the team. Those individuals, although they may share the goal of the medical good of a particular patient, are not devoted to the just and competent care of the patient population seen in the ED environment. Cooperative relationships with those outside the health care team are often conducive to better care for patients, but this is not always the case. Advocacy for the patient population or ED colleagues may require that adversarial roles be taken when the good of the ED patient population and the good of the ED health care team are in conflict with those outside the team.

Two examples will help explicate these tensions and their just resolution. Emergency medicine and surgical services often disagree on the management of abdominal pain in the ED. Emergency physicians often treat abdominal pain with narcotic medications, while surgeons contend that this impedes their diagnostic evaluation. The literature on this issue suggests that the use of narcotics is in the best interest of individual patients and results in more effective diagnostic investigations to determine whether surgery is necessary..$^{12} 13$ Emergency physicians have moral, medical, and legal responsibility for these patients whose primary complaint is pain. Surgeons treat these patients as potential surgical candidates. They do not assume moral responsibility for these patients unless they have a problem correctable by surgery. Therefore the goals of treatment are not shared by surgeons and EPs in these cases. Although building good relationships with other departments is helpful, treatment of pain is no longer a question of style, but of legality. EPs should distribute literature describing evidence based conclusions about treating abdominal pain to the surgical service and make clear how every EP will respond in these cases. ED professionals should not compromise methods of treatment which the evidence suggests is sound to flatter the egos of others in the hospital.

Patient management differences may result in physicians from different services acting in a hostile manner to those working in the ED, particularly nurses and medical residents. Procedures exist for addressing these clinical management differences, such as writing letters describing points of dispute to the chairperson or medical director. When those procedures are ignored, and other physicians attempt to use intimidation to express their anger, staff physicians and nursing administration in the ED should feel a proprietary interest in their staff and should zealously advocate for those in their department. These kinds of breaches of procedure or human decency should be dealt with on an immediate basis by the most senior person present, and in subsequent days should be the subject of conversation between the chairperson of the ED and the other involved department. Health care professionals who use physical intimidation in the ED in the form of throwing items, blocking doorways, or pushing should be banned from the ED.

While loyalty to employees is always important, it is vital in the often chaotic environment of the ED. Providers receive little appreciation from their anxious and suffering patients and their family members, and they risk injury from violent patients and other visitors. To ask them to tolerate these behaviours from other medical professionals would make their jobs intolerable. Emergency department employees need to know that they are valued members of a team, that they are respected as individuals, and that they work in an environment in which loyalty is valued and practiced.

\section{CONCLUSION}

A virtue ethic can be very appropriate in addressing ethical issues in the ED, but it must have as its ultimate telos the medical good of individual patients constrained by the wellbeing of the ED's patient population. Aristotle's vision of virtue always sought the good of the "body politic". Not only is this conception justifiable on ethical grounds, it fits the practice of EM much more naturally than a dyadic ethic.

When the medical good of the patient population is seen as the goal of EM, justice emerges as the most important virtue in resolving dilemmas. Providers should always demonstrate respect and loyalty for their coworkers and aim for the just allocation of all scarce resources in the ED, including money, time, patient rooms, and care giver energy. This model can be applied to most ethical issues in the ED. Its notion of justice builds on clinical practice skills already employed by EPs and yields effective patient care. (Although in this essay we have focused on EM, there is no reason to believe that this model could not also be effectively applied in other medical specialties.)

\section{Authors' affiliations}

J Girod, The Poynter Center for the Study of Ethics and American Institutions, Indiana University, Bloomington, Indiana, USA

A W Beckman, Department of Emergency Medicine, Indiana University, Bloomington, Indiana, USA

\section{REFERENCES}

1 Pellegrino ED. Professionalism, profession and the virtues of the good physician. Mt Sinai J Med 2002;69:378-84.

2 Siegler MA. Professional values in modern clinical practice. Hastings Cent Rep 2000;30(suppl 4): 19-22S.

3 Ludmerer KM. Instilling professionalism in medical education. JAMA 1999;282:830-2.

4 Shelton W. Can virtue be taught? Acad Med 1999;74:671-4

5 Adams J, Schmidt T, Sanders A, et al. Professionalism in emergency medicine. Acad Emerg Med 1998;5:1193-9.

6 Larkin GL, Binder L, Houry D, et al. Defining and evaluating professionalism: a core competency for graduate emergency medicine education. Acad Emerg Med 2002;9:1249-56.

7 Sanders AB, Keim SM, Sklar D, et al. Emergency physicians and the biomedical industry. Ann Emerg Med 1992;21:556-8.

8 Anon. Virtue in emergency medicine. Acad Emerg Med 1996;3:961-6.

9 Finkel MA, Adams JG. Professionalism in emergency medicine. Emerg Med Clin North Am 1999;17:443-50.

10 Pellegrino ED. The virtuous physician and the ethics of medicine. In: Shelp EE, ed. Virtue and medicine: explorations in the character of medicine. Norwell, MA: Kluwer Academic Publishers, 1985:243-55.

11 Simon JR, Dwyer J, Goldfrank LR. The difficult patient. Emerg Med Clin North Am 1999;17:353-70.

12 McHale PM, LoVecchio F. Narcotic analgesia in the acute abdomen: a review of prospective trials. Eur J Emerg Med 2001;8:131-6.

13 Pace S, Burke TF. Intravenous morphine for early pain relief in patients with acute abdominal pain. Acad Emerg Med 1996;3:1086-92. 